\title{
Editorial
}

\section{Of Form, Context, and Use}

Kirk St.Amant

\author{
Editor: Communication Design Quarterly \\ Louisiana Tech University \\ stamantk@latech.edu
}

\section{Form and Function}

Many of us have heard the expression "form follows function." It's a nice idea in theory: The notion the item we create inherently lends itself to a particular use. After all, the shape of a hammer should intrinsically tell us the tasks we can perform with that tool - be it pounding or pulling nails. But those of us who study human behavior related to use know this relationship of form to function is often far from the case. In truth, the connection between design and use is far more complex and often unpredictable.

In truth, it is context that generally drives what constitutes a functional form. That is, the context in which we use items often affects how we perceive them in terms of what tasks they can be used for and how well they can perform such activities in that setting. The hammer that might be useful in an open setting might be ineffective in an enclosed space where the user does not have the space to swing it with the force needed to pound in nails. As such, we communication designers need to continually reflect upon context any time we consider design in terms of use and usability.

\section{Context, Use, and Design}

Perhaps the first step in this process involves revising the language we use to discuss the relationship of form to function. Thus, the adage of "form follows function" needs to give way to "creation conforms to context." By linking the idea of setting to that of design, we create a mindset that recognizes design can not be universal (i.e., we can't create an item that will be equally usable in 
all contexts). We also realize the central role an understanding of context of use must play in any design process.

Interestingly, technologies are not completely at the mercy of environments when it comes to design and use. After all, humans regularly employ technologies to change the context in which they are used. (We can, for example, use a hammer to create a space that is ideally formed for using that hammer as designed.) This symbiotic relationship between creation and contexts means we, as communication designers, will always face the challenge of understanding new settings in relation to user needs and expectations. Thus, our research needs to examine the environments in which we employ technologies in communication practices. The entries in this issue of Communication Design Quarterly represent a collective exploration of such factors.

\section{Overview of the Issue}

The issue begins with two editorial that explore how the contexts in which we communicate affect how we share information and ideas. The first entry, Katherine Hepworth's "Big Data Visualization: Promises \& Pitfalls" examines the ways in which we think about and the approaches we use to convey large amounts of data via visual displays. In a second editorial, Beth L. Hewett asks us to rethink our approach to literacy to include the technologies we use to craft communiques and access information. In so doing, these two pieces help us realize the contexts in which we communicate and the factors we need to consider when addressing issue of usability.

These editorials are followed by four articles that further explore how setting can affect use. Heidi Skurat Harris and Michael Greer, for example, ask us to re-consider how the learning management technologies instructors use to interact with students in online classes have implications for how instruction is delivered. Amanda M. Licastro, in turn, builds on these ideas by exploring how student perspectives of the technologies used in online education affect perceptions of and expectations associated with online education. In this way, the combined ideas presented by Skurat Harris and Greer and by Licastro provide a holistic perspective of users involved in communities created by online educational contexts. 
This notion of community is further examined by Jennifer deWinter, Carly A. Kocurek, and Stephanie Vie who use a feminist approach to review the idea of labor (particularly social labor) as created within the context of gaming communities. Their entry helps readers better understand how perceptions of work guide activity in a community in ways that influence design in different settings. James KiwanukaTondo and Keon Pettiway expand this examination of context to the international level by reviewing how different geopolitical, historical, and cultural factors affect risk communication in the Greater Horn of Africa. Their review focuses on how the application of SWOT principles (Strengths, Weaknesses, Opportunities, and Threats) can enhance our understanding of design in such context.

The issue then concludes with two book reviews on texts that explore contextual aspects affecting ideas of use and usability. In the first entry, Daniel L. Hocutt reviews Ehren Pflugfelder's book Communicating Mobility and Technology: A Material Rhetoric for Persuasive Transportation. In so doing, Hocutt re-enforces how humans and technologies are interconnected in ways that allow each to influence the evolution of the other in terms of the contexts in which the two interact. Similarly, Ann Shivers-McNair's review of Risk Communication and Miscommunication: Case Studies in Science, Technology, Engineering, Government, and Community Organizations by Carolyn Boiarsky reminds us of how contextual factors affect perceptions of and communication about risk in different environments. By summarizing these ideas, these two reviews expand upon the relationship between context, design, and use noted in the earlier editorials and articles in this issue.

\section{Concluding Thoughts}

Usability is a complex concept requiring designers to understand a range of variables when creating materials. As such, the contexts that affect use become central area of focus in researching usability and addressing user expectations based on experience. By reviewing these connections, the entries in this issue provide insights into a range of dynamics affecting use across different contexts. Readers should thus view these editorials, articles, and reviews as examples of the kinds of factors to consider when examining usability in different settings. 